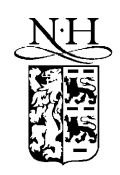

\title{
Perturbative approach to higher derivative and nonlocal theories
}

\author{
Tai-Chung Cheng, Pei-Ming Ho, Mao-Chuang Yeh \\ Department of Physics, National Taiwan University, Taipei 106, Taiwan, ROC \\ Received 6 December 2001; accepted 9 January 2002
}

\begin{abstract}
We review a perturbative approach to deal with Lagrangians with higher or infinite order time derivatives. It enables us to construct a consistent Poisson structure and Hamiltonian with only first time derivatives order by order in coupling. We show that, to the lowest order, the Hamiltonian is bounded from below whenever the potential is. We consider spacetime noncommutative field theory as an example. (c) 2002 Elsevier Science B.V. All rights reserved.
\end{abstract}

PACS: 11.10.Ef; 11.10.Lm

\section{Introduction}

In the canonical formulation, we usually consider Lagrangians with only first time derivatives. However, higher derivative theories, including nonlocal theories, also have many physical applications. For example, when one integrates out high energy degrees of freedom in a local field theory, the low energy effective action is generically nonlocal [1]. Higher derivative theories were considered even earlier in order to find a finite quantum field theory [2], before the advent of renormalization. Moreover, theories with infinite derivatives are inevitable from the viewpoint of string theory [3,4]. There are many other examples, such as higher derivative gravity [5], meson-nucleon interactions [6], and spacetime noncommutative field theory [7,8], and so on.

Unfortunately, an exact treatment of generic nonlocal theories is so far unknown. This was viewed as a serious problem for string theory [3]. However, in most cases, higher derivative terms appear as higher order corrections in the effective Lagrangian, so a perturbative approximation scheme would already be very useful.

E-mail address: pmho@phys.ntu.edu.tw (P.-M. Ho). 
In this paper we consider theories for which the free part of the Lagrangian involves only first time derivatives, but there can be higher derivatives to any (finite or infinite) order in the interaction terms. According to the canonical formulation, the phase space is $2 k$-dimensional for Lagrangians with $k$ th time derivatives [9]. For a low energy effective theory, however, we usually assume that there are as many degrees of freedom as the free part of the Lagrangian tells us. This is also an assumption for the S-matrix to be unitary in a quantum field theory [10]. Presumably the extra dimensions of the phase space are associated with certain high energy degrees of freedom which are integrated out from the description.

What we need is a formalism to deal with Lagrangians with finite or infinite derivatives without introducing new (high energy) degrees of freedom. It should also allow us to consistently construct Poisson brackets and Hamiltonian perturbatively order by order, and in the end give us an effective Lagrangian with only first time derivatives.

In [11] it was proposed to remove higher derivative terms in the Lagrangian by field redefinition. But it is unclear how to achieve this to arbitrary orders for a generic theory, or whether this is always possible. A general procedure which provides a Hamiltonian formulation order by order in the number of time derivatives in the interaction terms was constructed in [12]. The perturbative approach [3] that we will discuss in this paper is a modification of that. It is based on an expansion in the coupling constant, with no restriction to the number of derivatives in each order. This technique has been used extensively in [13] for higher derivative gravitational theory. Although this approach was studied in detail in [3], which we recommend the interested readers to refer to for related issues, such as the quantization or causality problems, we present this approach here in a slightly different fashion, with some derivations and proof explicitly given. In fact, we have discovered the same techniques independently, and were only acquainted with previous works on this subject by a referee after this work was finished.

What is the new progress we made on this subject? (1) We show that, to the lowest order, we can describe the result most simply by a change of variable. Remarkably, it turns out that, in terms of this new variable, the Hamiltonian is bounded from below whenever the potential is. (2) We give the formal proof that the perturbative approach can be carried out to all orders consistently. This proof also shows that if an exact solution to the equation of motion is known, the same approach can immediately give the final result. (3) In order to understand the difference between the original system and its perturbative formulation, we demonstrate by an example that when the calculation is carried out to all orders, the description of low energy modes is exact, including the effect of their interactions with high energy modes, but high energy degrees of freedom are still absent. (4) We apply this approach to field theories living on noncommutative spacetime, and comment on the spacetime uncertainty relation.

This paper is organized as follows. In Section 2, we briefly review the canonical formulation of theories with finite time derivatives, and explain the problems in dealing with infinite derivatives. We comment there and show in Appendix A that one-loop unitarity is not affected by introducing higher spatial derivatives, but is at stake when higher time derivatives are present. In Section 3 the perturbative approach for higher derivative theories is constructed. One can have a consistent Hamiltonian formulation of the theory to an arbitrary order in coupling. We also give a formal proof that this procedure can be 
consistently carried out to all orders. Finally, in Section 4 we give three examples. In the first example [3] we demonstrate explicitly how the perturbative approach works to the second order. The second example is used to show that this approach suppresses high energy degrees of freedom, but provides an exact description for the low energy modes. We also apply this approach to the example of spacetime noncommutative field theory.

\section{Canonical formulation}

\subsection{Lagrangians with finite time derivatives}

Here we review the canonical formulation for classical theories with finite time derivatives. Consider a Lagrangian with $k$ th time derivatives

$$
L_{0}=L_{0}\left(q, \dot{q}, \ddot{q}, \ldots, q^{(k)}\right),
$$

where $q^{(i)}$ is the $i$ th time derivative of $q$. Let us apply the formalism of [14]. The variation of the action

$$
S=\int_{t_{i}}^{t_{f}} d t L,
$$

with respect to $q$ is found to be the time integral of $\delta q$ times the Euler-Lagrange equation

$$
\sum_{n=0}^{k}\left(-\frac{d}{d t}\right)^{n} \frac{\partial L_{0}}{\partial q^{(n)}}=0,
$$

plus a boundary term

$$
\left[\sum_{i} P_{i} \delta q^{(i)}\right]_{t_{i}}^{t_{f}}
$$

where $P_{i}$ is the conjugate momentum of $q^{(i)}$

$$
P_{i}=\sum_{j=0}^{k-i-1}\left(-\frac{d}{d t}\right)^{j} \frac{\partial L_{0}}{\partial q^{(i-j+1)}} .
$$

The symplectic structure can be directly read off from this as

$$
\Omega=\sum_{i=0}^{k-1} d P_{i} d q^{(i)} .
$$

The Hamiltonian is

$$
H=\sum_{i=0}^{k-1} P_{i} q^{(i)}-L\left(q, \dot{q}, \ldots, q^{(k-1)}, q^{(k)}\right),
$$


where $q^{(k)}$ is viewed as a function of $q^{(i)}$ 's for $i=0, \ldots, k-1$ and $P_{k-1} \cdot{ }^{1}$ Since $H$ is linear in $P_{1}, \ldots, P_{k-2}$, the energy spectrum is unbounded from below.

Another approach to deal with higher derivative Lagrangians is to introduce new variables to replace higher derivatives of $q$, and use Lagrange multipliers to ensure that we have not introduced any new degrees of freedom. One natural choice is to define new variables $q_{i}$ by $q_{i}=q^{(i)}$ (Exercise 1.26 in [15]), and another is to set $q_{i}=q^{(2 i)}$. In any case, after introducing new variables, the Lagrangian only has first time derivatives. Canonical momenta of all variables, including the Lagrange multipliers, can be defined as usual. Some of these will impose primary constraints. Dirac's procedure for constrained quantization can then be used to obtain the same result. See [15] for a detailed description of the general procedure.

\subsection{Lagrangians with infinite time derivatives}

For a Lagrangian with infinite time derivatives $L_{0}(q, \dot{q}, \ldots)$, one may hope that a trick similar to the one for Lagrangians with finite derivatives will work. A natural guess is to define the new Lagrangian as [16-19]

$$
L=L_{0}(Q)+\int d \lambda \mu(\lambda)\left(\dot{Q}(\lambda)-Q^{\prime}(\lambda)\right)
$$

where $L_{0}(Q)$ is obtained from $L_{0}[q]$ by the replacement

$$
q(t) \rightarrow Q(t, 0),\left.\quad q^{(n)}(t) \rightarrow \frac{d^{n}}{d \lambda^{n}} Q(t, \lambda)\right|_{\lambda=0} .
$$

By $Q^{\prime}$ we mean $\frac{d}{d \lambda} Q$, and $\mu$ is a Lagrange multiplier. This implies that $Q(t, \lambda)=q(t+\lambda)$.

However, the Euler-Lagrange equations for this new Lagrangian $L$ is in fact different from the Euler-Lagrange equation for the original Lagrangian $L_{0}$. Further constraints, or boundary conditions, have to be imposed [16-19].

Instead of trying to resolve this problem, we give some general reasons why a naive extension of the idea for finite time derivatives has difficulties.

For a nondengerate Lagrangian with $k$ th time derivatives, the phase space is $2 k$-dimensional for a single variable $q$. Let us choose $q^{(i)}(i=0,1, \ldots, 2 k-1)$ to be the coordinates on the phase space. The symplectic 2-form (5) implies that the Poisson bracket

$$
\left(q^{(i)}, q^{(j)}\right)=0
$$

for any $i, j \leqslant k-1$. The bracket is nontrivial when $i \leqslant k-1$ and $j \geqslant k$ and vise versa.

When we take $k \rightarrow \infty$, naively, the phase space has coordinates $\left\{q^{(i)}\right\}_{i=0,1, \ldots, \infty}$, and (9) holds for any $i$ and $j$. The nontrivial part of Poisson brackets is now completely lost!

On the other hand, if we use $q^{(i)}$ and $P_{i}(i=0,1, \ldots, k-1)$ as the phase space variables, in the limit $k \rightarrow \infty$, the phase space has coordinates $\left\{q^{(i)}, P_{i}\right\}_{i=0,1, \ldots, \infty}$, which seems to have twice the dimension of the previous guess.

Another problem with infinite derivatives is the Cauchy problem [20]. For a Lagrangian with $k$ th derivative its equation of motion is a differential equation of order $2 k$. To

\footnotetext{
1 We assume that the Lagrangian is nondegenerate. This can always be achieved by adding total derivatives.
} 
determine the trajectory uniquely, the initial values of $q^{(i)}$ for $i=0,1, \ldots, 2 k-1$, have to be given. Naively, when $k \rightarrow \infty$, all $q^{(i)}$ 's have to be given as initial conditions, but they will completely determine the trajectory of $q$ via Taylor expansion even without using the equation of motion. This seems to suggest that for infinite $k$ the Euler-Lagrange equation should be imposed as a constraint on the space of initial conditions, which is equivalent to the phase space, and the evolution for a point in the phase space is trivial. This viewpoint is consistent with the vanishing of Poisson brackets mentioned above, and is similar to the proposal of earlier works [16-19].

Although it is desirable to have a general formalism to deal with theories with infinite derivatives. It is known that different cases can have very different properties. For some interesting examples see $[3,17]$. Probably different theories with infinite derivatives may have to be treated very differently.

Normally, one needs to understand the classical Hamiltonian formulation of a system before quantization can be done. In the operator formalism we replace the Poisson brackets by commutators. In the path integral formalism, we should start with

$$
\int D q D p e^{\int d t(p \dot{q}-H)}
$$

for a system with unconstrained conjugate variables $p, q$. The formula

$$
\int D q e^{\int d t L}
$$

may or may not be correct.

Given a Lagrangian with infinite derivatives, if one simply use (11) to define a quantum system, ${ }^{2}$ the violation of unitarity is observed in several examples [21]. It is tempting to interpret $Q(t, \lambda)$ in (7) as a chiral field on an open string, and an intriguing possibility is that for certain infinite derivative theories one can find a procedure to add new degrees of freedom for consistency which eventually leads to string theory.

In the next section we will review a perturbative approach for low energy effective theories with infinite time derivatives. This approach allows us to change the Lagrangian order by order to a new Lagrangian with only first time derivatives. It is then possible to quantize the theory without breaking unitarity. In Appendix A we also show that, with the free part of the Lagrangian unchanged, arbitrary spatial derivatives in the interaction terms will not spoil unitarity at one-loop level, which is sufficient for low energy effective theories. In particular, an immediate consequence is that noncommutative field theories with only spatial noncommutativity is unitary at one loop level.

\section{Perturbative approach}

In this section we review an approach to deal with higher derivative terms perturbatively. This approach works equally well for Lagrangians with finite or infinite order time derivatives.

\footnotetext{
2 The Hamiltonian formulation of [18] also leads to (11).
} 
In many applications, higher derivative terms appear in the Lagrangian as higher order corrections to a low energy effective theory. However, according to the canonical Hamiltonian formulation, when higher order terms are introduced, the dimension of the phase space increases, no matter how small the correction term is. Intuitively, this discrete jump in dimension does not seem to be compatible with the physical interpretation of the higher derivative interaction terms as a perturbative correction to the theory.

To resolve this apparent conceptual conflict, we note that the new dimensions of the phase space correspond to those degrees of freedom which are not accessible at low energies. If all we want is to have some knowledge about how these high energy states affect the behavior of the low energy modes, we can choose to restrict ourselves to the low energy degrees of freedom on the phase space. Therefore, the main idea behind this approach is to project the symplectic structure on the whole phase space onto the subspace of low energy states, and then induce the Hamiltonian formulation for the reduced phase space. This approach can also be generalized to cases in which it is preferred to work on a bigger phase space with finitely many higher time derivatives kept in the end.

It is sufficient to illustrate this approach by considering a $(0+1)$-dimensional theory with a general Lagrangian

$$
L=\frac{1}{2} \dot{q}^{2}-\frac{m}{2} q^{2}-g V(q, \dot{q}, \ddot{q}, \ldots) .
$$

To be definite, let us consider the cases with infinite order time derivatives. Under variation, the action is

$$
\delta S=-\int d t(\mathrm{EOM}) \delta q+\left[\sum_{k=0}^{\infty} P_{k} \delta q^{(k)}\right]_{t_{i}}^{t_{f}},
$$

where the equation of motion is

$$
\mathrm{EOM} \equiv \ddot{q}+m^{2} q+g \sum_{n=0}^{\infty}\left(-\frac{d}{d t}\right)^{n} \frac{\partial V}{\partial q^{(n)}}=0,
$$

and $P_{n}$ is the canonical momenta for $q^{(n)}$

$$
P_{k}=\dot{q} \delta_{k 0}-g \sum_{n=k+1}^{\infty}\left(-\frac{d}{d t}\right)^{n-k-1} \frac{\partial V}{\partial q^{(n)}} .
$$

The symplectic two-form is

$$
\Omega=\sum_{k=0}^{\infty} d P_{k} d q^{(k)}
$$

\subsection{First order approximation}

In an exact treatment, all $q^{(n)}$ 's are independent. In the low energy approximation, we only keep $q$ and $\dot{q}$. Using the equation of motion, we can replace higher derivatives of $q$ 
by lower derivatives. To the lowest order approximation,

$$
q^{(n)} \approx \begin{cases}\left(-m^{2}\right)^{n / 2} q & (n=\text { even }), \\ \left(-m^{2}\right)^{(n-1) / 2} \dot{q} & (n=\text { odd }) .\end{cases}
$$

The boundary term of $\delta S(13)$ reduces to

$$
\left[\Pi_{0} \delta q+\Pi_{1} \delta \dot{q}\right]_{t_{i}}^{t_{f}}=\left[\left(\dot{q}-g \xi_{0}\right) \delta q-g \xi_{1} \delta \dot{q}\right]_{t_{i}}^{t_{f}}
$$

where

$$
\begin{aligned}
& \xi_{0}=\sum_{k=0}^{\infty} \sum_{n=2 k+1}^{\infty}\left(-m^{2}\right)^{k}\left(-\frac{d}{d t}\right)^{n-2 k-1} \frac{\partial V}{\partial q^{(n)}}, \\
& \xi_{1}=\sum_{k=0}^{\infty} \sum_{n=2 k+2}^{\infty}\left(-m^{2}\right)^{k}\left(-\frac{d}{d t}\right)^{n-2 k-2} \frac{\partial V}{\partial q^{(n)}} .
\end{aligned}
$$

Note that $\xi_{0}$ and $\xi_{1}$ should be viewed as functions of $q$ and $\dot{q}$ via the replacement (17).

We find the symplectic two-form (16) as

$$
\Omega=d \Pi_{0} d q+d \Pi_{1} d \dot{q}=\left(-1+g \frac{\partial \xi_{0}}{\partial \dot{q}}-g \frac{\partial \xi_{1}}{\partial q}\right) d q d \dot{q} .
$$

It follows that the Poisson bracket should be

$$
(q, \dot{q}) \simeq 1+g \frac{\partial \xi_{0}}{\partial \dot{q}}-g \frac{\partial \xi_{1}}{\partial q}
$$

To the lowest order in $g$, the Poisson bracket can be written as

$$
(x, p)=1,
$$

where

$$
x=q+g \xi_{1}, \quad p=\dot{q}-g \xi_{0} .
$$

The Hamiltonian is

$$
H=\Pi_{0} \dot{q}+\Pi_{1} \ddot{q}-L,
$$

where we replace all higher derivatives of $q$ by (17). We find

$$
H=\frac{1}{2} p^{2}+\frac{m^{2}}{2} x^{2}+g \widetilde{V}(x, p)
$$

where $\widetilde{V}(x, p)=V\left(x, p,-m^{2} x, \ldots\right)$. Note that, if the potential $V$ is bounded from below, the first order Hamiltonian is also bounded from below.

One can check that the Hamilton equations

$$
\dot{q}=(q, H), \quad \ddot{q}=(\dot{q}, H)
$$

reproduce the equation of motion (14) to the first order in $g$. The corresponding Lagrangian is

$$
\tilde{L}=p \dot{x}-H=\frac{1}{2} \dot{x}^{2}-\frac{m^{2}}{2} x^{2}-g \widetilde{V}(x, \dot{x}) .
$$


Its Euler-Lagrange equation agrees with the original system to the first order in $g$. Since the final expression only contains first derivatives, its quantization is straightforward.

\subsection{Higher order approximation}

For higher order corrections, we first iterate the equation of motion (14) up to a certain $\operatorname{order} \mathcal{O}\left(g^{n}\right)$. For example, at the first order,

$$
q^{(2)} \rightarrow-m^{2} q-g \sum_{n=0}^{\infty}\left(-\frac{d}{d t}\right)^{n} \frac{\partial V}{\partial q^{(n)}},
$$

where we should also use the replacement (17) to change the last term into a function of $q$ and $\dot{q}$ only. Higher derivatives of $q$ can also be replaced by functions of $q$ and $\dot{q}$ up to the same order in $g$ by differentiating with respect to time and repeatedly using (29) as

$$
q^{(n)} \approx \begin{cases}\left(-m^{2}\right)^{n / 2} q-g \sum_{l=1}^{n / 2} \sum_{k=0}^{\infty}\left(-m^{2}\right)^{n / 2-l}\left(\frac{d}{d t}\right)^{k+l-1} \frac{\partial V}{\partial q^{(k)}} & (n=\text { even }), \\ \left(-m^{2}\right)^{(n-1) / 2} \dot{q}-g \sum_{l=1}^{(n-1) / 2} \sum_{k=0}^{\infty}\left(-m^{2}\right)^{(n-1) / 2-l}\left(\frac{d}{d t}\right)^{k+l-1} \frac{\partial V}{\partial q^{(k)}} & (n=\text { odd }) .\end{cases}
$$

In general, we can have all $q^{(i)}$ 's expressed as a function of $q$ and $\dot{q}$ only, up to a certain order $\mathcal{O}\left(g^{n}\right)$. This helps us to derived the symplectic form from (16)

$$
\Omega=\sum_{i} d P_{i} d q^{(i)}=d \Pi_{0} d q+d \Pi_{1} d \dot{q}=\left(-\frac{\partial \Pi_{0}}{\partial \dot{q}}+\frac{\partial \Pi_{1}}{\partial q}\right) d q d \dot{q} .
$$

The final Hamiltonian is defined by (25) with all higher derivatives of $q$ replaced by functions of $q, \dot{q}$. Its Hamilton equations (27) gives the equation of motion up to $\mathcal{O}\left(g^{n+1}\right)$.

An effective Lagrangian can be defined as

$$
\tilde{L}=p \dot{x}-H
$$

for a conjugate pair $(x, p)=1$. For such Lagrangians we do not expect violation of unitarity since it contains only first time derivatives. Although we expect such theories to be nonrenormalizable in the usual (power-counting) sense, it remains to be seen whether it is renormalizable in the modern sense [22].

\subsection{To all orders: a formal proof}

Now we give a formal proof for the self-consistency of the perturbative formulation. From the equation of motion (14), in principle one can find

$$
\ddot{q}=f(q, \dot{q})
$$

for a certain function $f$ to all orders in $g$ by iteration (or inspiration). Higher derivatives of $q$ can be derived from this

$$
q^{(n)}=f_{n}(q, \dot{q}),
$$


where the functions $f_{n}$ can be obtained recursively

$$
f_{n+1}=\left[\frac{d}{d t} f_{n}\right]=\frac{\partial f_{n}}{\partial q} \dot{q}+\frac{\partial f_{n}}{\partial \dot{q}} f,
$$

where we used the notation

$$
\left.[A] \equiv A\right|_{q^{(n)}=f_{n}} .
$$

Here are a few identities that will come in handy in the following. From (15), we find

$$
\dot{P}_{k}=\frac{\partial L_{0}}{\partial q^{(k)}}-P_{k-1} .
$$

For an arbitrary function $A$ on the phase space, we also find the following identities

$$
\begin{aligned}
& \frac{d}{d t}[A]=[\dot{A}]+\frac{\partial[A]}{\partial \dot{q}}(\ddot{q}-f), \\
& \frac{\partial[A]}{\partial q}=\sum_{k=0}^{\infty}\left[\frac{\partial A}{\partial q^{(k)}}\right] \frac{\partial f_{k}}{\partial q},
\end{aligned}
$$

where one can also replace $\partial / \partial q$ by $\partial / \partial \dot{q}$ in the last formula.

The variables $\Pi_{0}, \Pi_{1}$ can be read off from

$$
\Pi_{0} \delta q+\Pi_{1} \delta \dot{q}=\sum_{k=0}^{\infty} P_{k} \delta q^{(k)},
$$

and we find

$$
\begin{aligned}
& \Pi_{0}=\sum_{k=0}^{\infty}\left[P_{k} \frac{\partial q^{(k)}}{\partial q}\right], \\
& \Pi_{1}=\sum_{k=0}^{\infty}\left[P_{k} \frac{\partial q^{(k)}}{\partial \dot{q}}\right] .
\end{aligned}
$$

The Hamiltonian is

$$
H=\left[\Pi_{0} \dot{q}+\Pi_{1} \ddot{q}-L\right] .
$$

The Hamilton equations (27) based on the symplectic structure (31) are

$$
\begin{aligned}
& \dot{\Pi}_{0}+\Pi_{1} \frac{\partial f}{\partial q}-\frac{\partial \Pi_{1}}{\partial q}(\ddot{q}-f)-\frac{\partial\left[L_{0}\right]}{\partial q}=0, \\
& \dot{\Pi}_{1}+\Pi_{0}+\Pi_{1} \frac{\partial f}{\partial \dot{q}}-\frac{\partial \Pi_{1}}{\partial \dot{q}}(\ddot{q}-f)-\frac{\partial\left[L_{0}\right]}{\partial \dot{q}}=0 .
\end{aligned}
$$

With the help of (37)-(39), one can show from (41), (42) that (45) is automatically satisfied, and that (44) is equivalent to the equation of motion (33).

Certainly, (33) is not the only solution of the equation of motion (14). It may be extended to a more general solution with many free parameters

$$
\ddot{q}=f\left(q, \dot{q}, c_{1}, c_{2}, \ldots\right) \text {. }
$$


These extra parameters are associated with high energy degrees of freedom. Ignoring them, or setting them to zeros corresponds to taking the low energy limit in which these high energy modes are "minimally excited". That is, they are only excited to be consistent with the equation of motion, but we do not consider their independent degrees of freedom. We will consider a system of coupled springs in the next section as an example to show this property more concretely.

\section{Examples}

\subsection{An example of second order approximation}

In this section we show the perturbative procedure explicitly for an example to the second order. Consider the Lagrangian [3]

$$
L=\frac{1}{2} \dot{q}^{2}+\frac{a}{2} q^{2}+g q \ddot{q}^{2} .
$$

Its Euler-Lagrange equation is

$$
\ddot{q}=a q+g \ddot{q}^{2}+2 g(q \ddot{q})^{\cdot .} .
$$

Following the procedure outlined in the previous section, we find

$$
\Pi_{0}=\dot{q}-2 g(q \ddot{q})^{\cdot}, \quad \Pi_{1}=2 g q \ddot{q}
$$

from (41), (42). Iterating (48) to the first order in $g$, we find

$$
\ddot{q}=a q+g\left(5 a^{2} q^{2}+4 a \dot{q}^{2}\right)+\mathcal{O}\left(g^{2}\right) .
$$

Taking derivatives, we get

$$
\begin{aligned}
& q^{(3)}=a \dot{q}+g\left(18 a^{2} q \dot{q}\right)+\mathcal{O}\left(g^{2}\right), \\
& q^{(4)}=a^{2} q+g\left(23 a^{3} q^{2}+22 a^{2} \dot{q}^{2}\right)+\mathcal{O}\left(g^{2}\right),
\end{aligned}
$$

and so on.

Up to third order terms in $g$, we find

$$
\begin{aligned}
& \Pi_{0}=\dot{q}-4 g(a q \dot{q})-2 g^{2}\left(23 a^{2} q^{2} \dot{q}+4 a \dot{q}^{3}\right), \\
& \Pi_{1}=2 g\left(a q^{2}\right)+2 g^{2}\left(5 a^{2} q^{3}+4 a q \dot{q}^{2}\right) .
\end{aligned}
$$

The Poisson bracket is

$$
(q, \dot{q})=1+8 g a q+4 g^{2}\left(35 a^{2} q^{2}+8 a \dot{q}^{2}\right)+\mathcal{O}\left(g^{3}\right) .
$$

The Hamiltonian is

$$
\begin{aligned}
H= & \frac{1}{2} \dot{q}^{2}-\frac{a}{2} q^{2}+g\left(a^{2} q^{3}-4 a q \dot{q}^{2}\right)+2 g^{2}\left(5 a^{3} q^{4}-19 a^{2} q^{2} \dot{q}^{2}-4 a \dot{q}^{4}\right) \\
& +\mathcal{O}\left(g^{3}\right)
\end{aligned}
$$


One can check that the Hamilton equations (27) give the equation of motion (48) correct to the third order in $g$.

The effective Lagrangian is

$$
\tilde{L}=p \dot{q}-H,
$$

where $p$ is the phase space variable conjugate to $q$, i.e., $(q, p)=1$. From $(54)$, we can solve for $p$

$$
p=\dot{q}-8 g a q \dot{q}-4 g^{2}\left(19 a^{2} q^{2} \dot{q}+\frac{8}{3} a \dot{q}^{3}\right)+\mathcal{O}\left(g^{3}\right) .
$$

Thus we find the Lagrangian for $q, \dot{q}$

$$
\begin{aligned}
\tilde{L}= & \frac{1}{2} \dot{q}^{2}+\frac{a}{2} q^{2}-g\left(a^{2} q^{3}+4 a q \dot{q}^{2}\right)+2 g^{2}\left(-5 a^{3} q^{4}-19 a^{2} q^{2} \dot{q}^{2}-\frac{4}{3} a \dot{q}^{4}\right) \\
& +\mathcal{O}\left(g^{3}\right) .
\end{aligned}
$$

The Euler-Lagrange equation derived from this Lagrangian is

$$
\ddot{q}=a q+g\left(5 a^{2} q^{2}+4 a \dot{q}^{2}\right)+4 g^{2}\left(19 a^{3} q^{3}+35 a^{2} q \dot{q}^{2}\right)+\mathcal{O}\left(g^{3}\right) .
$$

This is exactly what one gets from (48) by iteration, in the same way we got (50), but to a higher order correction in $g$.

\subsection{Coupled springs}

Consider two springs $A$ and $B$ of spring constants $k$ and $K$, respectively, as in Fig. 1. One end of $A$ is fixed to a wall. The other end of $A$ is connected to $B$, with a mass $M$ attached to the joint. Another mass $m$ is attached to the end of $B$.

For $K \gg k$ and $M \sim m$, the degrees of freedom of $M$ is much harder to excite than $m$. Let the coordinates of $m$ and $M$ be $x$ and $y$. The equations of motion are

$$
\begin{aligned}
& m \ddot{x}+k(x-y)=0, \\
& M \ddot{y}+K y+k(y-x)=0 .
\end{aligned}
$$

They imply that

$$
a x^{(4)}+b \ddot{x}+c x=0
$$

where $a=M m, b=(M+m) k+m K, c=K k$. The effective Lagrangian for this equation of motion is

$$
L \propto \frac{1}{2}\left(-a \ddot{x}^{2}+b \dot{x}^{2}-c x^{2}\right) .
$$

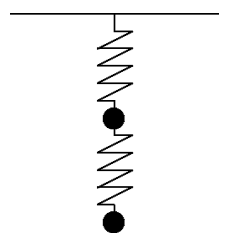

Fig. 1. 
Assume that $K \gg k$, then the first term is negligible for small $\ddot{x}$. Imagine that someone studies the behavior of the spring system at low energies by conducting experiments in which only the variable $x$ is manipulated, and finds an approximate Lagrangian as (63) without the first term. The prediction of the natural frequency is

$$
\omega_{0}^{2}=\frac{K k}{(M+m) k+m K} \simeq \frac{k}{m}\left(1-\frac{k}{K} \frac{M+m}{m}\right)
$$

to the lowest order in $\mathcal{O}(k / K)$.

Now suppose that via more accurate experiments, the first term in the Lagrangian (63) is put in. When the perturbative approach is carried out to the first order, one finds

$$
\omega^{2} \simeq \frac{k}{m}\left(1-\frac{k}{K}\right)
$$

This is a better approximation than (64) to the real natural frequency. In this case we can even ask what will we get by carrying out the perturbative approach to all orders. It is straightforward to see that the answer is

$$
\omega^{2}=\frac{1}{2 a}\left(b-\sqrt{b^{2}-4 a c}\right) .
$$

This is precisely one of the two normal mode frequencies of the exact equation of motion. Although it does not tell us anything about the other natural frequency at higher energy $\omega^{2} \simeq K / M$, it describes the exact effect of the stiff spring on the low energy mode.

In this example, we see that the physical meaning of reducing the phase space to the space of $q, \dot{q}$ in the perturbative formulation is to suppress the excitation of high energy modes in a way consistent with the exact evolution, and only to allow low energy modes to be excited.

\subsection{Spacetime noncommutative field theory}

Spacetime noncommutative field theories are interesting and important for many reasons [23]. Although the naive quantization is non-unitary and non-causal [23], perhaps a proper treatment will lead to the noncommutative open string (NCOS) theory [23,24].

The exact treatment of this system is outside the scope of this paper. We will only study this theory perturbatively. The zeroth order results are in fact already studied in [25], where it was shown that the kinetic term with or without the $*$-product results in the same Hamiltonian formulation.

Consider the Lagrangian

$$
L=\frac{1}{2}\left(\partial_{\mu} \phi \partial_{\mu} \phi-m^{2} \phi^{2}\right)+\lambda \phi * \phi * \phi .
$$

The $*$-product is defined by

$$
f * g(x)=\left.e^{\frac{1}{2} \theta^{\mu \nu} \partial_{\mu} \partial_{\nu}^{\prime}} f(x) g\left(x^{\prime}\right)\right|_{x^{\prime}=x} .
$$

It makes no difference whether we put $*$-products in the quadratic terms. 
For simplicity, consider the $(1+1)$-dimensional case

$$
[t, x]_{*}=\theta .
$$

As we saw in Section 3.1, by a field redefinition, the effective Lagrangian is given by (28) at the lowest order. For the case at hand it is

$$
\tilde{L} \simeq \frac{1}{2}\left(\partial_{\mu} \Phi \partial_{\mu} \Phi-m^{2} \Phi^{2}\right)+\lambda \Phi \bar{*} \Phi \bar{*} \Phi,
$$

where the $\bar{*}$-product is defined as $*$ in (68) with the replacement

$$
\partial_{t}^{n} \rightarrow \begin{cases}\left(-\partial_{x}^{2}+m^{2}\right)^{n / 2} & (n=\text { even }), \\ \left(-\partial_{x}^{2}+m^{2}\right)^{(n-1) / 2} \partial_{t} & (n=\text { odd }) .\end{cases}
$$

In the action defined by (70), one only needs $\partial_{t}^{n}$-terms for even $n$.

This result clarifies a conceptual puzzle. On one hand, the uncertainty relation

$$
\Delta t \Delta x \geqslant|\theta|,
$$

which follows from (69), implies that when we treat $t$ as a classical number, $\Delta x \rightarrow \infty$. Thus we should not associate independent physical degrees of freedom at different values of $x$. It suggests a reduction of the number of degrees of freedom [26,27]. On the other hand, higher derivatives in the action imply a larger phase space in canonical formulation. These two intuitions seem to contradict each other.

This puzzle may now be understood as follows. The uncertainty relation (72) does not really follow from (69), because the time $t$, unlike $x$, is not an operator in quantum mechanics. However, according to (71) and the definition of $\bar{*}$-product, it is an effective description of the fact that the extent of spatial nonlocality (the shift in $x$ for $\Phi$ in the interaction term) is proportional to the energy scale, which is approximated by the free energy $\left(-\partial_{x}^{2}+m^{2}\right)^{1 / 2}$ for small $\lambda$. Incidentally, this relation is reminiscent of the characteristic property of strings [28]. This is also one of the reasons why spacetime noncommutative field theories are of interest to us. On the other hand, in an exact treatment, the consistent high energy completion of spacetime noncommutative theory is presumably the NCOS theory, which contains a lot more degrees of freedom.

\section{Acknowledgements}

The authors thank Marc Henneaux, Miao Li, Feng-Li Lin, John Wang for helpful discussions. This work is supported in part by the National Science Council, the CosPA project of the Ministry of Education, the National Center for Theoretical Sciences, Taiwan, ROC and the Center for Theoretical Physics at National Taiwan University.

\section{Appendix A. One-loop unitarity for derivative interactions}

Consider a scalar field for example. In $D$-dimensional spacetime, its propagator is

$$
\Delta(x)=\theta\left(x_{0}\right) \Delta^{+}(x)+\theta\left(-x_{0}\right) \Delta^{-}(x)
$$




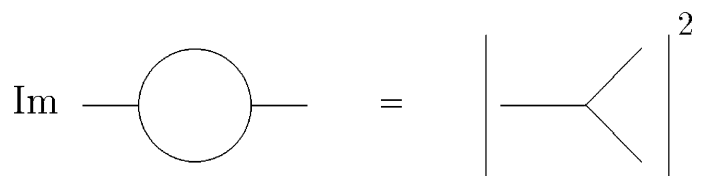

Fig. 2.

where

$$
\Delta^{ \pm}(x)=\frac{1}{(2 \pi)^{D-1}} \int d^{D} k \delta\left(k^{2}+m^{2}\right) \theta\left( \pm k_{0}\right) e^{i k \cdot x} .
$$

The left-hand side of the diagram in Fig. 2 is

$$
\begin{aligned}
\widetilde{\Sigma}\left(x_{f}-x_{i}\right)= & i g^{2} F\left(\partial_{f}^{(1)}, \partial_{f}^{(2)}\right) F\left(\partial_{i}^{(1)}, \partial_{i}^{(2)}\right) \\
& \times\left.\Delta_{1}\left(x_{f}^{(1)}-x_{i}^{(1)}\right) \Delta_{2}\left(x_{f}^{(2)}-x_{i}^{(2)}\right)\right|_{x^{(1)}=x^{(2)}=x},
\end{aligned}
$$

where the interaction is modified by a (Hermitian) function $F$ of derivatives of two particles. For the special case of interactions in noncommutative field theories,

$$
F\left(\partial^{(1)}, \partial^{(2)}\right)=e^{\frac{i}{2} \theta^{\mu \nu} \partial_{\mu}^{(1)} \partial_{\nu}^{(2)}}
$$

By Fourier transform,

$$
\Sigma(p)=\left.i g^{2} \int d^{D} x e^{-i p \cdot x} F(\partial) F(-\partial) \Delta_{1}\left(x^{(1)}\right) \Delta_{2}\left(x^{(2)}\right)\right|_{x^{(1)}=x^{(2)}=x} .
$$

If there is no time derivative in $F$, we find

$$
\begin{aligned}
\operatorname{Im}(\Sigma(p))=\frac{g^{2}}{2} \int \frac{d^{D-1} k_{1} d^{D-1} k_{2}}{(2 \pi)^{D-2}} \frac{F\left(i k_{1}, i k_{2}\right)}{2 \omega\left(k_{1}\right)} \frac{F\left(-i k_{1},-i k_{2}\right)}{2 \omega\left(k_{2}\right)} \delta^{D-1}\left(p-k_{1}-k_{2}\right) \\
\times\left(\delta\left(p_{0}-\omega_{1}\left(k_{1}\right)-\omega_{2}\left(k_{2}\right)\right)+\delta\left(p_{0}+\omega_{1}\left(k_{1}\right)+\omega_{2}\left(k_{2}\right)\right)\right),
\end{aligned}
$$

which is exactly the statement of unitarity. The same derivation can be extended to other one-loop diagrams, the point being that spatial coordinates do not have to be explicitly integrated over in the calculation. Thus we see that interaction terms with spatial derivatives preserve unitarity. The derivation breaks down when $F$ contains time derivatives. The unitarity was found to be preserved for like-like noncommutativity in [29].

\section{References}

[1] A.O. Barvinsky, C.A. Vilkovisky, Nucl. Phys. B 282 (1987) 163; A.O. Barvinsky, C.A. Vilkovisky, Nucl. Phys. B 333 (1990) 471.

[2] A. Pais, G.E. Uhlenbeck, Phys. Rev. 79 (1950) 145.

[3] D.A. Eliezer, R.P. Woodard, Nucl. Phys. B 325 (1989) 389.

[4] H. Hata, Phys. Lett. B 217 (1989) 438;

H. Hata, Nucl. Phys. B 329 (1990) 698. 
[5] K.S. Stelle, Phys. Rev. D 16 (1977) 953;

J. Julve, M. Tonin, Nuovo Cimento B 46 (1978) 137.

[6] P. Kristensen, C. Moller, K. Dan, Vidensk. Selsk. Mat.-Fys. Medd. 27 (1952) 7.

[7] A. Connes, M.R. Douglas, A. Schwarz, JHEP 9802 (1998) 003, hep-th/9711162; N. Seiberg, E. Witten, JHEP 9909 (1999) 032, hep-th/9908142.

[8] N. Seiberg, L. Susskind, N. Toumbas, JHEP 0006 (2000) 044, hep-th/0005015.

[9] M. Ostrogradski, Mem. Acad. St. Petersbourg VI (4) (1850) 385.

[10] S. Weinberg, The Quantum Theory of Fields I, Cambridge Univ. Press, Cambridge, 1995, Sections 3.1, 3.2.

[11] D. Barua, S.N. Gupta, Phys. Rev. D 16 (1977) 413.

[12] X. Jaén, J. Llosa, A. Molina, Phys. Rev. D 34 (1986) 2302.

[13] J.Z. Simon, Phys. Rev. D 41 (1990) 3720; J.Z. Simon, Phys. Rev. D 43 (1991) 3308.

[14] L.D. Faddeev, R. Jackiw, Phys. Rev. Lett. 60 (1988) 1692.

[15] M. Henneaux, C. Teitelboim, Quantization Of Gauge Systems, Princeton Univ. Press, Princeton, 1992.

[16] J. Llosa, J. Vives, J. Math. Phys. 35 (1994) 2856.

[17] R.P. Woodard, Phys. Rev. A 62 (2000) 052105, hep-th/0006207.

[18] J. Gomis, K. Kamimura, J. Llosa, Phys. Rev. D 63 (2001) 045003, hep-th/0006235.

[19] K. Bering, A note on non-locality and Ostrogradski's construction, hep-th/0007192.

[20] D.L. Bennett, H.B. Nielsen, R.P. Woodard, Phys. Rev. D 57 (1998) 1167, hep-th/9707088.

[21] J.L. Barbon, E. Rabinovici, Phys. Lett. B 486 (2000) 202, hep-th/0005073;

J. Gomis, T. Mehen, Nucl. Phys. B 591 (2000) 265, hep-th/0005129;

M. Chaichian, A. Demichev, P. Presnajder, A. Tureanu, Eur. Phys. J. C 20 (2001) 767, hep-th/0007156; L. Alvarez-Gaume, J.L. Barbon, R. Zwicky, JHEP 0105 (2001) 057, hep-th/0103069;

T. Mateos, A. Moreno, Phys. Rev. D 64 (2001) 047703, hep-th/0104167;

A. Bassetto, L. Griguolo, G. Nardelli, F. Vian, JHEP 0107 (2001) 008, hep-th/0105257.

[22] J. Gomis, S. Weinberg, Nucl. Phys. B 469 (1996) 473, hep-th/9510087.

[23] N. Seiberg, L. Susskind, N. Toumbas, JHEP 0006 (2000) 021, hep-th/0005040.

[24] R. Gopakumar, J. Maldacena, S. Minwalla, A. Strominger, JHEP 0006 (2000) 036, hep-th/0005048; R. Gopakumar, S. Minwalla, N. Seiberg, A. Strominger, JHEP 0008 (2000) 008, hep-th/0006062.

[25] R. Amorim, J. Barcelos-Neto, Remarks on the canonical quantization of noncommutative theories, hep-th/ 0108186.

[26] P.M. Ho, M. Li, Nucl. Phys. B 596 (2001) 259, hep-th/0004072;

P.M. Ho, M. Li, Nucl. Phys. B 590 (2000) 198, hep-th/0005268.

[27] M. Li, Dimensional reduction via noncommutative spacetime: bootstrap and holography, hep-th/0103107.

[28] T. Yoneya, in: K. Kawarabayashi, A. Ukawa (Eds.), Wandering in the Fields, World Scientific, 1987, p. 419; M. Li, T. Yoneya, Phys. Rev. Lett. 78 (1997) 1219, hep-th/9611072.

[29] O. Aharony, J. Gomis, T. Mehen, JHEP 0009 (2000) 023, hep-th/0006236. 